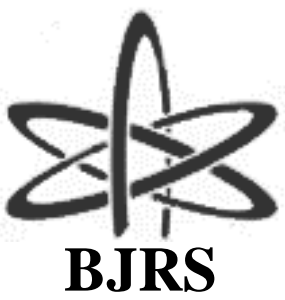

\author{
BRAZILIAN JOURNAL \\ $\mathrm{OF}$ \\ RADIATION SCIENCES \\ 05-02 (2017) 01-07
}

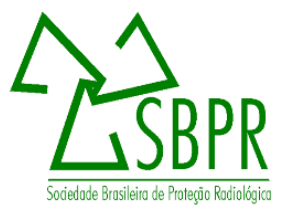

\title{
Estimativa das incertezas correlacionadas aos testes de desempenho de ativímetros
}

\author{
C.H.S. Sousa ${ }^{\mathrm{a}, \mathrm{b}}$; G.J. Teixeira ${ }^{\mathrm{a}}$; L.G. Padilha ${ }^{\mathrm{b}, \mathrm{c}}$; G.S. Araujo ${ }^{\text {c; J.G.P. Peixoto }}{ }^{\mathrm{a}}$ \\ ${ }^{a}$ Serviço de Metrologia das Radiações Ionizantes, LNMRI/IRD?CNEN, 22783-127, Rio de Janeiro-RJ, Brasil \\ .${ }^{b}$ Serviço de Radiologia, HUCFF/UFRJ, 21941-913, Rio de Janeiro-RJ, Brasil. \\ ${ }^{c}$ Fundação Tecnico-Educacional Souza Marques,21310-310 Rio de Janeiro-RJ, Brasil. \\ chenrique@ird.gov.br
}

\begin{abstract}
RESUMO
Ativímetros devem ser submetidos a testes de desempenho para a verificação da funcionalidade conforme recomendações técnicas e normativas. Este estudo verificou a correlação entre duas metodologias para a medição das grandezas independentes radiação de fundo e fonte-padrão referência certificada e rastreada. As metodologias basearam-se no protocolo indicado no documento técnico da Agência Internacional de Energia Atômica e nos limites normativos adotados pela legislação brasileira. A grandeza independente radiação de fundo apresentou uma variação dependente da metodologia aplicada na medição, e o resultado apresentou um coeficiente de correlação verdadeiro, possibilitando melhorar a estimativa das incertezas associadas em 3,43\%.
\end{abstract}

Palavras-chave: ativímetro, incerteza, câmara de ionização.

\begin{abstract}
Activimeters must be submitted to performance tests to verify functionality according to the techniques and regulations. This study verified the correlation between two methodologies for a measurement of the independent quantities, as background radiation and a certified reference source. The methodologies were based on protocol indicated on technical document of the International Atomic Energy Agency and the regulatory limits adopted by the Brazilian legislation. An independent magnitude of background radiation presented a dependent variation of the applied methodology in the measurement, and the result presented a true correlation coefficient, allowing an estimate of the associated uncertainties in $3.43 \%$.
\end{abstract}

Keywords: activimeter, uncertainty, ionization chamber. 


\section{INTRODUÇÃO}

A metrologia, além de estimar grandezas, controla equipamentos conforme especificações técnicas ou limites normativos. A apresentação de resultados sem parâmetros metrológicos, como rastreabilidade de padrões ou determinação das incertezas associadas pode comprometer qualquer conclusão fundamentada nos resultados da série de medições. De acordo com as recomendações técnicas da Agência Internacional de Energia Atômica - AIEA, toda instrumentação utilizada em medicina nuclear deve passar periodicamente por testes de desempenho para a verificação da qualidade dos resultados obtidos (1).

$\mathrm{O}$ instrumento que mede a atividade (A) de amostras radioativas administradas em pacientes submetidos à terapia ou procedimentos diagnósticos em medicina nuclear é denominado ativímetro, calibrador de doses ou ainda curiômetro. É constituído basicamente por uma câmara de ionização (CI) selada, onde é gerada uma corrente de ionização, sendo esta processada e exibida por uma eletrônica associada. Sua calibração é realizada pelo fabricante utilizando fontes-padrão referência, certificadas e rastreadas, para determinar a curva de calibração para os diversos radionuclídeos (2). A resposta característica deste instrumento depende intrinsecamente da natureza e da pressão do gás, do material e da espessura da parede da câmara. Outros fatores podem, ainda, influenciar o resultado das medições, tais como, as diferentes energias e taxas de emissões de fótons (3).

A qualidade dos resultados emitidos por estes instrumentos é determinada por testes de desempenho, cujos valores das medições são comparados aos valores referência, determinando os percentuais de variação em relação aos valores verdadeiros convencionais(4,5). Entretanto, os protocolos utilizados nas medições não são explícitos quanto às formas de execução, podendo levar o operador a obter resultados distintos entre duas metodologias distintas.

Duas grandezas são consideradas não correlacionadas quando as variações aleatórias de uma delas não influenciam as variações aleatórias da outra. Caso isto não aconteça, elas são denominadas correlacionadas ou estatisticamente dependentes. A estimativa da incerteza padrão combinada em medições diretas considera importante a verificação da correlação, pois, o resultado da medição pode influenciar o valor da incerteza. 
Desta forma, este trabalho procurou verificar se havia correlação entre as duas metodologias para a obtenção dos resultados do teste de exatidão, determinando a significância na escolha da forma de entrada dos dados das duas grandezas distintas durante a medição, para então determinar as incertezas associadas aos testes, terminando por explicitar seu impacto no resultado final.

\section{MATERIAIS E MÉTODOS}

Foram utilizados três ativímetros, cujas características principais estão descritas na tabela 1. Para os testes de exatidão e precisão foi utilizada uma fonte-padrão referência de ${ }^{57} \mathrm{Co}$, selada e certificada, rastreada pelo National Institute of Standards and Technology -NIST. O teste de linearidade utilizou um frasco de vidro para eluição e o radionuclídeo ${ }^{99 \mathrm{~m}} \mathrm{Tc}$, obtido através da eluição de um gerador de tecnécio, ambos fornecidos pelo Instituto de Pesquisas Energéticas e Nucleares (IPEN/SP).

Tabela 1: Características mais relevantes dos ativímetros utilizados neste estudo.

\begin{tabular}{lccc}
\hline \multirow{2}{*}{ CARACTERÍSTICAS } & \multicolumn{3}{c}{ CALIBRADORES } \\
& \multicolumn{1}{c}{ A e B } & C \\
\hline Faixa (MBq) & min & $7,0 \times 10^{-4}$ & $3,7 \times 10^{-1}$ \\
& max & $7,0 \times 10^{5}$ & $3,7 \times 10^{4}$ \\
Resolução (MBq) & & 0,001 & 0,370 \\
Exatidão (\%) & $\pm 2,0$ & $\mathrm{Ni}^{\mathrm{a}}$ \\
Precisão (\%) & $\pm 0,1$ & $\mathrm{Ni}^{\mathrm{a}}$ \\
Linearidade (\%) & $\pm 2,0$ & 3,4 \\
Tipo de detector & $\mathrm{CI}^{b}$ & $\mathrm{GM}^{c}$ \\
\hline${ }^{a}$ Não informado & & \\
${ }^{b}$ Câmara de ionização & &
\end{tabular}

As medições tiveram como referência o Serviço de Metrologia de Radionuclídeos - SEMRA, do Laboratório Nacional de Metrologia das Radiações Ionizantes LNMRI/IRD/CNEN. A metodologia aplicada aos testes de desempenho foi definida e recomendada pelo Technical Report Series $n^{\circ} 454$ da AIEA (6).

Para o teste de exatidão foi estudado se as metodologias, sequencial e intercalada, das medições da radiação de fundo e da fonte padrão referência produziam resultados distintos e dependentes. Para isso, foram realizadas dez medições sequenciais da radiação de fundo e dez da fonte-padrão 
referência, com intervalos de 15 segundos entre cada medição. Posteriormente, foram realizadas dez medições da radiação de fundo e da fonte-padrão referência de modo intercalado. Todos os testes de desempenho seguiram os protocolos descritos nas recomendações da AIEA, sendo que, para o teste de linearidade foi aplicado o método de decaimento da amostra.

A correlação entre duas grandezas independentes é considerada não correlacionada, quando as variações aleatórias de uma delas não influenciam as variações aleatórias da outra, caso contrário elas são ditas correlacionadas. A determinação da correlação é necessária para a verificação do grau de correlação, pois, pode influenciar expressivamente o valor de uma incerteza de medição.

Neste estudo foram correlacionadas duas metodologias de medição propostas, determinando as médias e os desvios padrões das séries de medição e aplicado o momento de Pearson (equação 1), onde, $x_{i}$ corresponde às medições independentes da fonte padrão- referência, $\bar{x}$ a média aritmética destas medições, $y_{i}$ as medições independentes da radiação de fundo e $\bar{y}$ a média aritmética de suas medições (7).

$$
r_{x y}=\frac{\left(\left(\sum_{i=1}^{n} x_{i} y_{i}\right)-\left(\frac{\left(\sum_{i=1}^{n} x_{i}\right)\left(\sum_{i=1}^{n} y_{i}\right)}{n}\right)\right)}{\sqrt{\left(\sum_{i=1}^{n}\left(x_{i}-\bar{x}\right)^{2}\left(\sum_{i=1}^{n} y_{i-1}-\right)^{2}\right)}}
$$

Uma vez estabelecida a correlação, determinou-se a variância combinada das grandezas de entrada $x_{i}$, e $y_{i}$, sendo estas tipo A $(8,9)$, conforme equação 2 ,

$$
S(\bar{x}, \bar{y})=\left(\frac{1}{n(n-1)}\right) \sum_{i=1}^{n}\left(x_{i}-\bar{x}\right)\left(y_{i}-\bar{y}\right)
$$

A avaliação das incertezas fundamentou-se nas recomendações do Guia Para a Expressão da Incerteza de Medição (10), considerando como incerteza tipo A, o desvio padrão percentual das séries de medições dos testes de exatidão $\left(\sigma_{e}\right)$ e radiação de fundo $\left(\sigma_{f}\right)$. As incertezas tipo B, 
vislumbraram todas as outras contribuições que não foram consideradas repetitividade, como a precisão $\left(\sigma_{\mathrm{p}}\right)$, a reprodutibilidade $\left(\sigma_{\mathrm{r}}\right)$, a linearidade $\left(\sigma_{\mathrm{L}}\right)$, a resolução $\left(\sigma_{\mathrm{res}}\right)$, as impurezas radionuclídicas da fonte-padrão $\left(\sigma_{\mathrm{i}}\right)$, o volume $\left(\sigma_{\mathrm{v}}\right)$, e a incerteza declarada no certificado de calibração da fonte-padrão referência.

\section{RESULTADOS}

Os testes normativos de desempenho realizados nos três ativímetros apresentaram resultados dentro dos limites recomendados pela literatura, conforme apresentados na tabela 3. Durante as medições foi verificado, que os valores da radiação de fundo aumentaram sensivelmente, durante o método de medição intercalado (tabela 2), evidenciando que esta grandeza de entrada é influenciada pela medição da fonte padrão-referência.

Tabela 2: Resultados percentuais dos testes de desempenho normativos.

\begin{tabular}{lccc}
\hline \multicolumn{1}{c}{ Tipo de } & \multicolumn{3}{c}{ Calibradores } \\
Medição & $\mathbf{A}$ & $\mathbf{B}$ & $\mathbf{C}$ \\
\hline Direta & $0,02 \%$ & $0,03 \%$ & $0,12 \%$ \\
Intercalada & $0,04 \%$ & $0,06 \%$ & $0,32 \%$ \\
\hline
\end{tabular}

O resultado deste estudo apresentou correlação verdadeira, com variância (fator de correlação), inversa e fracamente positiva para os calibradores de dose A e B e direta e fortemente positiva para o calibrador de doses C (tabela 4). Os resultados da estimativa das incertezas padrão combinadas e expandidas, para os testes de desempenho não correlacionadas (NC) e correlacionadas (C) aplicados para cada um dos três ativímetros, são apresentados na tabela 4, assim como o nível de confiança.

Tabela 3: Resultados percentuais dos testes de desempenho normativos.

\begin{tabular}{lccc}
\hline \multicolumn{1}{c}{ Testes } & \multicolumn{3}{c}{ Calibradores } \\
& A & B & C \\
\hline Radiação de Fundo & $0,08 \%$ & $0,64 \%$ & $0,99 \%$ \\
Linearidade & $2,0 \%$ & $3,1 \%$ & $6,8 \%$ \\
Reprodutibilidade & $0,16 \%$ & $0,14 \%$ & $0,64 \%$ \\
Precisão & $0,22 \%$ & $0,21 \%$ & $1,24 \%$ \\
Exatidão & $0,10 \%$ & $0,14 \%$ & $2,08 \%$ \\
\hline
\end{tabular}


Tabela 4: Resultados percentuais da correlação e da variância entre as grandezas independentes fonte padrão-referência $\left(x_{i}\right)$ e radiação de fundo $\left(y_{i}\right)$.

\begin{tabular}{lccc}
\hline & \multicolumn{3}{c}{ Calibradores } \\
& A & B & C \\
\hline Correlação & $-0,0104 \%$ & $-0,0040 \%$ & $0,099 \%$ \\
Variância & $0,008 \%$ & $0,014 \%$ & $0,457 \%$ \\
\hline
\end{tabular}

Tabela 5: Valores das estimativas das incertezas não correlacionadas (NC) e C correlacionadas (C), dos testes para a fonte padrão-referência.

\begin{tabular}{ccccccc}
\hline & \multicolumn{3}{c}{ Calibrador A } & \multicolumn{2}{c}{ Calibrador B } & \multicolumn{2}{c}{ Calibrador C } \\
& NC & C & NC & C & NC & C \\
\hline $\boldsymbol{u}$ & 2,004 & 2,001 & 3,006 & 3,001 & 8,838 & 7,124 \\
$\boldsymbol{U}$ & 4,00 & 4,00 & 6,01 & 6,00 & 17,68 & 14,25 \\
$\boldsymbol{k}$ & 2,00 & 2,00 & 2,00 & 2,00 & 2,03 & 2,00 \\
\hline \multicolumn{3}{l}{ * Nível de confiança de 95,45\%. }
\end{tabular}

\section{CONCLUSÕES}

Entre as duas metodologias aplicadas aos três ativímetros, constata-se a existência de correlação entre a medição direta e intercalada das grandezas radiação de fundo e fonte padrão-referência, uma vez que $r\left(x_{i}, y_{i}\right)$ é diferente de zero e se encontra entre $-1 \mathrm{e}+1$. Para os ativímetros $\mathrm{A}$ e $\mathrm{B}$, a correlação foi inversa e fracamente negativa, pois $r\left(x_{i}, y_{i}\right)$ se apresentou menor e distante a zero com valores negativos, e para o ativímetro $\mathrm{C}$, a correlação foi direta e positiva, pois, $r\left(x_{i}, y_{i}\right)$, conforme apresentado na tabela 4 , apresentou valores positivos mais próximos a 1. Conclui-se então, que a correlação entre as duas grandezas independentes é verdadeira independente das metodologias de medição aplicadas, com resultados para os ativímetros $\mathrm{A}$ e $\mathrm{B}$, sem implicações práticas, porém, para o ativímetro $\mathrm{C}$ a correlação permitiu melhorar a incerteza expandida em 3,43\%, permitindo inferir que, caso outras grandezas de entrada sejam correlacionadas, os resultados finais podem sofrer alterações metrológicas significativas. 


\section{REFERÊNCIAS}

1. International Atomic Energy Agency IAEA. Quality Assurance for Radioactivity Measurement in Nuclear Medicine [Internet]. October. 2006. Available from: http://www-pub.iaea.org/MTCD/publications/PDF/TRS454_web.pdf

2. Sousa CHS, Teixeira GJ, Peixoto JGP. Estimated of the associated uncertaintes with the linearity test of activimeters. J Phys [Internet]. 2015;575:12028. Available from: http://stacks.iop.org/1742-

6596/575/i=1/a=012028?key=crossref.a5dee393760d3297187de5c72dccac85

3. Schrader H. Activity Measurements with ionization chambers. Bureau International des Poids et Mesures; 1997.

4. CNEN. REQUISITOS DE SEGURANÇA E PROTEÇÃO RADIOLÓGICA. CNEN-3.05 Brasil: Comissão Nacional de Energia Nuclear; 2013 p. 26.

5. ANVISA. RESOLUÇÃO DA DIRETORIA COLEGIADA - RDC No 38, DE 4 DE JUNHO DE 2008. Brasilia; 2008 p. 15.

6. IAEA. Quality Assurance for Radioactive Measurements in Nuclear Medicine. IAEA Library Cataloguing in Publication Data. Viena; 2006.

7. Mendenhall W. Probabilidade e Estatística. 1 ${ }^{\circ}$. Campus, editor. 1985. 277 p.

8. Peixoto JGP, Almeida CE de. CÂMARA DE AR LIVRE COMO PADRÃO PRIMÁRIO DA GRANDEZA EXPOSIÇÃO PARA FEIXES DE RAIOS-X ( 80 - 280 KVp). Revista Brasileira de Engenharia. Rio de Janeiro; 1990;VOL. 7 N.:351-8.

9. Inmetro. Vocabulário internacional de termos fundamentais e gerais de metrologia. [Internet]. 2007. 10. Inmetro. GUM 2008 - Guia para a expressão de incerteza de medição. 1a edição. Rio de Janeiro; 2012. 141 p. 